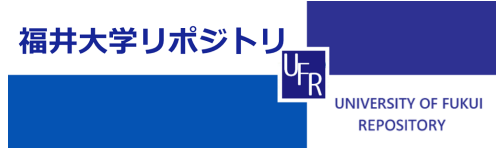

On phase patterntransition in star-coupl ed Wen- bri dge oscill at or s wi th par anet er devi at i ons

\begin{tabular}{|l|l|}
\hline $\begin{array}{l}\text { jour nal or } \\
\text { publ i cat i on titl e }\end{array}$ & $\begin{array}{l}\text { Proc. of the 2005 Eur opean Conf er ence on } \\
\text { Ci r cuit Theory and Desi gn (ECCTD' 05) }\end{array}$ \\
\hline vol une & 2005 \\
\hline page range & Paper I D268 \\
\hline year & 2005 \\
\hline URL & ht t p: //hdl . handl e. net /10098/2947 \\
\hline
\end{tabular}




\section{On Phase Pattern Transition in Star-Coupled Wien-Bridge Oscillators with Parameter Deviations}

\author{
Seiichiro Moro *
}

\begin{abstract}
In this study, we investigate the basins of attraction of the phase patterns and clarify the effect of parameter deviations in pulse-driven star-coupled Wien-bridge oscillators with parameter deviations. From the simulation results, it is shown that some phase pattern can be seen easily and the others can be hardly seen because of the deviations. Moreover, the reachability problems of the phase patterns suggests that these systems can treat not only the spatial phase patterns but the spatio-temporal patterns.
\end{abstract}

\section{INTRODUCTION}

There have been many investigations of mutual synchronization and multimode oscillation in coupled oscillators [1]-[3]. In particular, we have reported synchronization phenomena observed from $N$ oscillators with the same natural frequency mutually coupled by one resistor [2,3]. In LC oscillators systems, we have confirmed that $N$-phase oscillation can be stably excited when each oscillator has strong nonlinearity [2]. In this case, there exist $(N-1)$ ! stable phase states according to the initial states. Moreover, we have investigated the coupled system with RC Wien-bridge oscillators. This system is suitable for VLSI implementation because the system does not include any inductors. They also exhibit the "phase-shift synchronization" and we can get $3^{N-1}$ different stable phase patterns [3]. Because these "star-coupled" oscillators exhibit a large number of different steady states, they would be used as a structural element of large scale memories and neural networks.

When we use the coupled oscillators systems as neural networks and large scale memories, it should be an important problem how to control the systems to get the appropriate phase patterns. To achieve the phase pattern control, we have proposed the star-coupled system of Wien-bridge oscillators driven by the periodic pulse train and confirmed that the stimulation of the pulse train can cause the phase pattern switching [4]. In this system, however, only the phase of the oscillator where the pulse train is directly added switches. Moreover, we have proposed two types of star-coupled Wien-bridge oscillators whose driving methods with pulse train are different [5]. In these systems, though multiple oscillators' phases can be switched by pulse train, there are

\footnotetext{
${ }^{*}$ Department of Electrical and Electronics Engineering, University of Fukui, Japan, e-mail: moro@ppc8100. fuee.fukui-u.ac.jp, tel.: +81-776-27-8762, fax: +81-776-27-8749.

${ }^{\dagger}$ Department of Electrical and Electronics Engineering, Fukui University of Technology, Japan, e-mail: matsu@ccmails.fukui-ut.ac.jp, tel.: +81-776-22-8111 (ext. 2563), fax: +81-776-29-7891.
}

\author{
Tadashi Matsumoro ${ }^{\dagger}$
}

some disadvantages in each system. To avoid these problems, we have proposed the star-coupled systems with some parameter deviations [6]. In these systems, the phase pattern switching of the successive multiple oscillators can be achieved due to the deviations. In such systems, it is considered that the symmetry of the system is collapsed by the parameter deviation. In this study, we calculate the basins of attraction of the phase patterns in the star-coupled Wien-bridge oscillators system stimulated by pulse using SPICE from some initial patterns. From the results, we show that not every pattern can derive from a certain initial pattern. However, the reachability problems of the phase patterns from one to another suggest that these systems can treat the spatio-temporal patterns. From these features, these will be convenient for the use as some kinds of neural networks and associative memories.

\section{CIRCUIT MODELS}

The circuit models are shown in Fig. 1. In this study, we propose the following two models.

Model 1 The switch unit is connected to Osc 4 .

Model 2 The switch unit is connected to the coupling resistor $r$.

In each model, the switch unit stimulates the starcoupled Wien-bridge oscillators. In this case, the switch closes $\Delta t$ seconds in every $T$ seconds, and the periodic pulse stimulation with period $T$ is added to the system. $T$ should be sufficiently large to achieve the synchronization within the period. The construction of the subcircuits is shown in Fig. 1 (c).

In both systems, the parameter deviations are provided by the different capacitance in each $C_{1} \sim C_{4}$. The capacitance $C_{k}$ is described as follows,

$$
C_{k}=C+(k-1) \Delta C
$$

where $C$ is the capacitance of the capacitor in subcircuit and $\Delta C$ is the deviation parameter. If $\Delta C$ is larger, the difference of the natural frequency of each oscillator becomes larger.

\section{SIMULATION RESULTS}

In this section, we show the simulation results in proposed models by standard circuit simulator package 


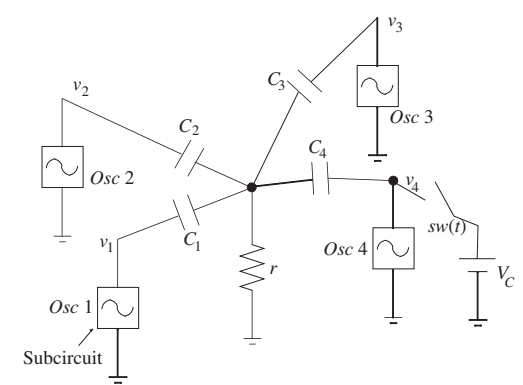

(a)

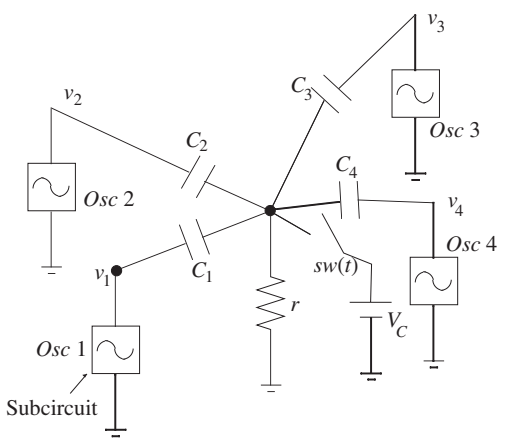

(b)

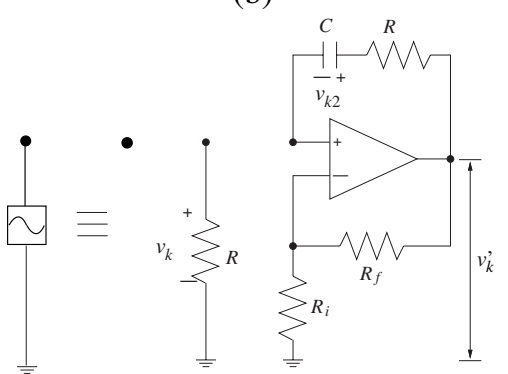

(c)

Osc 1 Osc 2

Ocs 3 Osc 4

(d)

Figure 1: Circuit models. (a) Switch unit is connected to an oscillator (Model 1). (b) Switch unit is connected to the coupling resistor (Model 2). (c) Construction of subcircuit. (d) Schematic of the system.

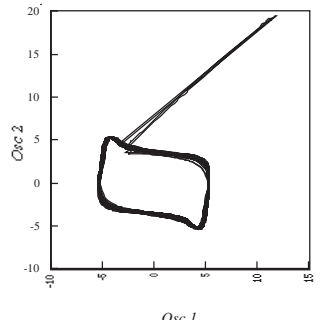

(a)

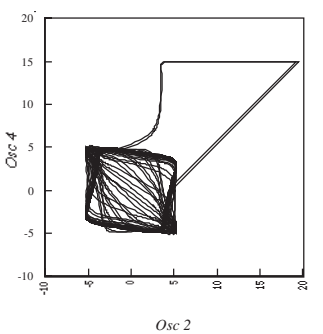

(c)

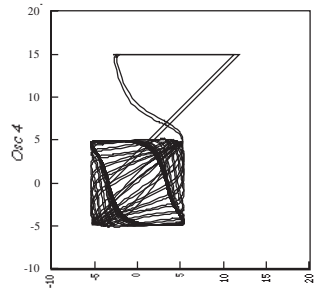

(b)
Figure 2: Lissajours' figures for $\Delta C=10^{-3} \mu \mathrm{F}$ for Model 1.

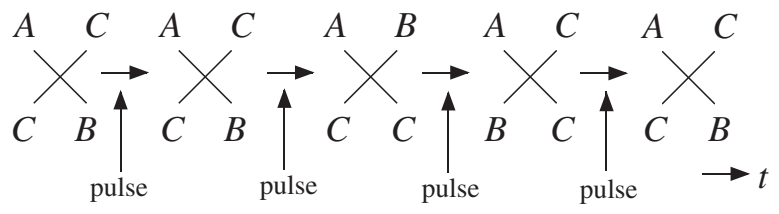

Figure 3: An example of the phase pattern transition when $\Delta C=10^{-3} \mu \mathrm{F}$ for Model 1 .

SPICE. In this study, we use the following circuit parameters: $R=10 \mathrm{k} \Omega, C=0.015 \mu \mathrm{F}, r=200 \Omega$, $R_{f}=14.7 \mathrm{k} \Omega, R_{i}=4.7 \mathrm{k} \Omega, \Delta t=50 \mu \mathrm{sec}, T=100 \mathrm{msec}$. In the following results, $A, B$ and $C$ indicate in-phase, $+120^{\circ}$ and $-120^{\circ}$ phase shift with respect to the phase of $O s c 1$, respectively.

Figures 2 and 3 show the results for Model 1 and Figure 4 shows the results for Model 2 when $\Delta C=10^{-3} \mu \mathrm{F}$. In these models, we can see successive phase pattern switching of the multiple oscillators due to the parameter deviations. From the results, it is shown that the in-phase synchronization of $O s c 1$ and $O s c 4$ is hardly seen. It is considered that this is because the natural frequencies of these oscillators are more different than the other combinations of the oscillators. Such parameter deviations make the system asymmetric and they affect the system dynamics and the derived phase patterns.

Next, we show the precise results of phase pattern switching. In the following results, the possible $27=$ $3^{4-1}$ phase patterns are indicated as the notation from $a$ to $z$ and $I P$ as shown in Fig. 5. In this case, $\Delta C=$ 


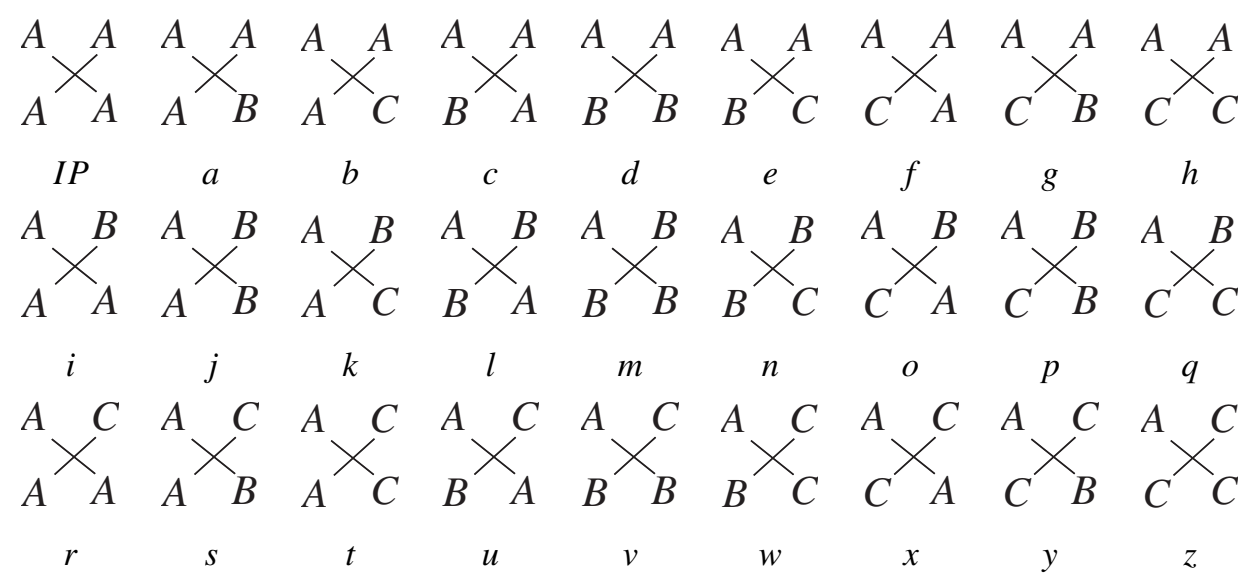

Figure 5: The notation of the phase patterns.

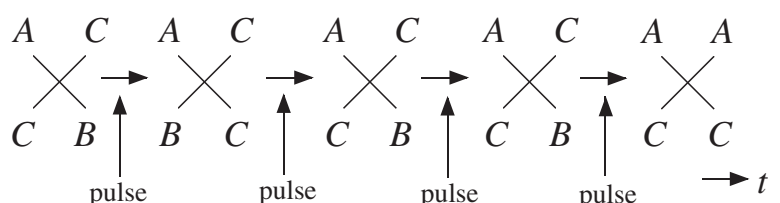

Figure 4: An example of the phase pattern transition when $\Delta C=10^{-3} \mu \mathrm{F}$ for Model 2 .

$10^{-3} \mu \mathrm{F}$ and the single pulse (i.e., not periodic) is added to the system. Tables $1-6$ show the phase patterns when the timing of the pulse, pulse voltage and the initial patterns are changed. In these cases, the phase patterns before adding the pulse are $h, v, w$ and $y$. In each model, not so many patterns appear after the pulses are added. In particular, in Table 3, only $q$ and $y$ are seen, therefore, some patterns cannot be derived directly from $y$. In such cases, the desired pattern has to be derived through the other patterns. For example, assume that we want the pattern $q$ from the initial pattern $v$. However, from Table 2 , there is no direct way to pattern $q$ from $v$. Therefore, we have to derive it by twice pulse stimulation, such that $v \rightarrow y \rightarrow q$, according to the tables. From this fact, the reachability problems from a pattern to another pattern should be important to use these systems as neural networks. From the tables show above, we can derive the directed graphs as shown in Fig. 6 which denote the transitions of the phase patterns. We can discuss the reachability problem using the incidence matrices of these graphs. On the other hand, the pattern sequences such as $v \rightarrow y \rightarrow q$ can be also patterns derived from the systems. In this case, phase patterns $a-z$ and $I P$ can be said as spatial pattens and sequence from patten to pattern can be said as temporal patterns. From this point of view, we can treat the spatio-temporal patterns in these systems and the possibility of these systems as
Table 1: 2 Parameter Phase Pattern Diagram for Model 1 (Initial Pattern $h$ ).

\begin{tabular}{|c||ccccccccccc|}
\hline \multicolumn{11}{|c||}{} & \multicolumn{11}{|c|}{ Phase } \\
\hline$V_{c}$ & 0 & $\frac{1}{5} \pi$ & $\frac{2}{5} \pi$ & $\frac{3}{5} \pi$ & $\frac{4}{5} \pi$ & $\pi$ & $\frac{6}{5} \pi$ & $\frac{7}{5} \pi$ & $\frac{8}{5} \pi$ & $\frac{9}{5} \pi$ \\
\hline \hline 15 & $y$ & $h$ & $h$ & $q$ & $h$ & $h$ & $h$ & $y$ & $y$ & $y$ \\
14 & $y$ & $w$ & $h$ & $w$ & $h$ & $h$ & $h$ & $y$ & $y$ & $y$ \\
13 & $y$ & $y$ & $h$ & $w$ & $h$ & $h$ & $h$ & $y$ & $y$ & $y$ \\
12 & $y$ & $y$ & $h$ & $h$ & $h$ & $h$ & $h$ & $w$ & $y$ & $y$ \\
11 & $y$ & $y$ & $h$ & $h$ & $h$ & $h$ & $h$ & $w$ & $w$ & $y$ \\
10 & $y$ & $y$ & $h$ & $h$ & $h$ & $h$ & $h$ & $h$ & $w$ & $y$ \\
\hline
\end{tabular}

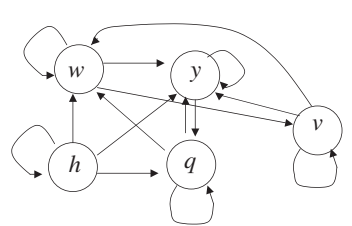

(a)

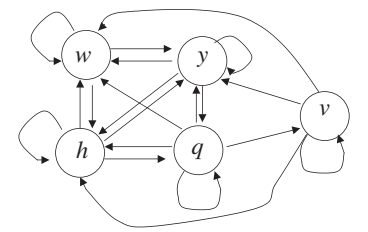

(b)
Figure 6: Directed graphs for the phase pattern transitions. (a) Model 1. (b) Model 2.

neural networks and memories will be expanded.

\section{CONCLUSIONS}

In this paper, we show the frequency of appearance of the phase patterns in pulse-driven star-coupled Wienbridge oscillators with the parameter deviations. The symmetry of the system is collapsed by such parameter deviations, and they affect the system dynamics and the phase patterns. From the results, it is shown that some phase pattern can be seen easily and that some phase patterns can be hardly seen because of the devia- 
Table 2: 2 Parameter Phase Pattern Diagram for Model 1 (Initial Pattern $v$ ).

\begin{tabular}{|c||cccccccccc|}
\hline \multicolumn{1}{|c||}{} & \multicolumn{11}{|c|}{ Phase } \\
\hline$V_{c}$ & 0 & $\frac{1}{5} \pi$ & $\frac{2}{5} \pi$ & $\frac{3}{5} \pi$ & $\frac{4}{5} \pi$ & $\pi$ & $\frac{6}{5} \pi$ & $\frac{7}{5} \pi$ & $\frac{8}{5} \pi$ & $\frac{9}{5} \pi$ \\
\hline \hline 15 & $v$ & $v$ & $v$ & $w$ & $w$ & $w$ & $w$ & $w$ & $y$ & $y$ \\
14 & $v$ & $v$ & $v$ & $w$ & $w$ & $w$ & $w$ & $w$ & $y$ & $y$ \\
13 & $v$ & $v$ & $v$ & $w$ & $w$ & $w$ & $w$ & $w$ & $y$ & $y$ \\
12 & $y$ & $v$ & $v$ & $w$ & $w$ & $w$ & $w$ & $w$ & $w$ & $y$ \\
11 & $y$ & $v$ & $v$ & $w$ & $w$ & $w$ & $w$ & $w$ & $w$ & $y$ \\
10 & $y$ & $v$ & $v$ & $v$ & $w$ & $w$ & $w$ & $w$ & $w$ & $y$ \\
\hline
\end{tabular}

Table 3: 2 Parameter Phase Pattern Diagram for Model 1 (Initial Pattern $y$ ).

\begin{tabular}{|c||cccccccccc|}
\hline \multicolumn{110}{|c|}{ Phase } \\
\hline$V_{c}$ & 0 & $\frac{1}{5} \pi$ & $\frac{2}{5} \pi$ & $\frac{3}{5} \pi$ & $\frac{4}{5} \pi$ & $\pi$ & $\frac{6}{5} \pi$ & $\frac{7}{5} \pi$ & $\frac{8}{5} \pi$ & $\frac{9}{5} \pi$ \\
\hline \hline 15 & $y$ & $y$ & $y$ & $q$ & $q$ & $q$ & $q$ & $q$ & $y$ & $y$ \\
14 & $y$ & $y$ & $y$ & $q$ & $q$ & $q$ & $q$ & $q$ & $y$ & $y$ \\
13 & $y$ & $y$ & $y$ & $q$ & $q$ & $q$ & $q$ & $q$ & $y$ & $y$ \\
12 & $y$ & $y$ & $y$ & $q$ & $q$ & $q$ & $q$ & $q$ & $q$ & $y$ \\
11 & $y$ & $y$ & $y$ & $q$ & $q$ & $q$ & $q$ & $q$ & $q$ & $y$ \\
10 & $y$ & $y$ & $y$ & $q$ & $q$ & $q$ & $q$ & $q$ & $q$ & $y$ \\
\hline
\end{tabular}

tions. Such phenomena can separate the preferred patterns from undesirable patterns. Moreover, the reachability problems of the phase patterns suggest that these systems can treat the spatio-temporal patterns. From these features, these will be convenient for the use as some kinds of neural networks and associative memories.

\section{References}

[1] H. Kimura and K. Mano, "Some properties of mutually synchronized oscillators coupled by resis-

Table 4: 2 Parameter Phase Pattern Diagram for Model 2 (Initial Pattern $h$ ).

\begin{tabular}{|c|c|c|c|c|c|c|c|c|c|c|}
\hline & \multicolumn{10}{|c|}{ Phase } \\
\hline$V_{c}$ & 0 & $\frac{1}{5} \pi$ & $\frac{2}{5} \pi$ & $\frac{3}{5} \pi$ & $\frac{4}{5} \pi$ & $\pi$ & $\frac{6}{5} \pi$ & $\frac{7}{5} \pi$ & $\frac{8}{5} \pi$ & $\frac{9}{5} \pi$ \\
\hline 15 & $w$ & $h$ & $w$ & $w$ & $w$ & $h$ & $h$ & $h$ & $w$ & $w$ \\
\hline 14 & $w$ & $h$ & $w$ & $w$ & $w$ & $h$ & $h$ & $h$ & $h$ & $w$ \\
\hline 13 & $w$ & $h$ & $w$ & $w$ & $w$ & $h$ & $h$ & $h$ & $w$ & $w$ \\
\hline 12 & $q$ & $h$ & $w$ & $w$ & $w$ & $h$ & $h$ & $h$ & $w$ & $w$ \\
\hline 11 & $q$ & $w$ & $w$ & $w$ & $w$ & $h$ & $h$ & $h$ & $h$ & $w$ \\
\hline 10 & $q$ & $h$ & $y$ & $w$ & $w$ & $h$ & $h$ & $h$ & $h$ & $q$ \\
\hline
\end{tabular}

Table 5: 2 Parameter Phase Pattern Diagram for Model 2 (Initial Pattern $v$ ).

\begin{tabular}{|c|c|c|c|c|c|c|c|c|c|c|}
\hline & \multicolumn{10}{|c|}{ Phase } \\
\hline$V_{c}$ & 0 & $\frac{1}{5} \pi$ & $\frac{2}{5} \pi$ & $\frac{3}{5} \pi$ & $\frac{4}{5} \pi$ & $\pi$ & $\frac{6}{5} \pi$ & $\frac{7}{5} \pi$ & $\frac{8}{5} \pi$ & $\frac{9}{5} \pi$ \\
\hline 15 & $y$ & $v$ & $v$ & $\bar{h}$ & $h$ & $w$ & $w$ & $h$ & $w$ & $w$ \\
\hline 14 & $y$ & $v$ & $v$ & $h$ & $h$ & $w$ & $w$ & $h$ & $w$ & $w$ \\
\hline 13 & $y$ & $v$ & $v$ & $h$ & $h$ & $w$ & $w$ & $h$ & $w$ & $w$ \\
\hline 12 & $y$ & $v$ & $v$ & $h$ & $h$ & $w$ & $w$ & $h$ & $w$ & $w$ \\
\hline 11 & $y$ & $w$ & $y$ & $h$ & $h$ & $w$ & $w$ & $v$ & $v$ & $w$ \\
\hline 10 & $y$ & $w$ & $h$ & $h$ & $h$ & $w$ & $w$ & $v$ & $v$ & $y$ \\
\hline
\end{tabular}

Table 6: 2 Parameter Phase Pattern Diagram for Model 2 (Initial Pattern $y$ ).

\begin{tabular}{|c||ccccccccccc|}
\hline \multicolumn{1}{|c||}{} & \multicolumn{11}{|c|}{ Phase } \\
\hline$V_{c}$ & 0 & $\frac{1}{5} \pi$ & $\frac{2}{5} \pi$ & $\frac{3}{5} \pi$ & $\frac{4}{5} \pi$ & $\pi$ & $\frac{6}{5} \pi$ & $\frac{7}{5} \pi$ & $\frac{8}{5} \pi$ & $\frac{9}{5} \pi$ \\
\hline \hline 15 & $y$ & $y$ & $h$ & $q$ & $q$ & $y$ & $y$ & $y$ & $w$ & $y$ \\
14 & $w$ & $y$ & $h$ & $h$ & $q$ & $y$ & $y$ & $y$ & $w$ & $y$ \\
13 & $y$ & $y$ & $h$ & $y$ & $q$ & $y$ & $y$ & $y$ & $w$ & $y$ \\
12 & $w$ & $w$ & $h$ & $y$ & $q$ & $y$ & $y$ & $y$ & $w$ & $y$ \\
11 & $y$ & $w$ & $h$ & $y$ & $q$ & $y$ & $y$ & $y$ & $w$ & $y$ \\
10 & $w$ & $w$ & $h$ & $y$ & $y$ & $y$ & $y$ & $y$ & $w$ & $y$ \\
\hline
\end{tabular}

tance," Trans. IECE, Vol. 48, No. 10, pp. 16471656, Oct. 1965 (in Japanese).

[2] S. Moro, Y. Nishio, and S. Mori, "Synchronization phenomena in oscillators coupled by one resistor," IEICE Trans. Fundamentals, Vol. E78-A, No. 2, pp. 244-253, Feb. 1995.

[3] S. Moro, Y. Nishio, and S. Mori, "Synchronization phenomena in RC oscillators coupled by one resistor," IEICE Trans. Fundamentals, Vol. E78-A, No. 10, pp. 1435-1439, Oct. 1995.

[4] S. Moro and T. Matsumoto, "Phase pattern switching in star-coupled Wien-bridge oscillators driven by pulse train,' Proc. of IEEE ISCAS '02, Vol. 5, pp. 313-316, May 2002.

[5] S. Moro and T. Matsumoto, "Driving methods for multiple oscillators' control in pulse-driven starcoupled Wien-bridge oscillators," Proc. of ECCTD '03, Vol. 1, pp. 306-309, Sept. 2003.

[6] S. Moro and T. Matsumoto, "Phase pattern switching in pulse-driven star-coupled Wien-bridge oscillators with parameter deviations," Proc. of RISP International Workshop on Nonlinear Circuits and Signal Processing, pp.475-478, Mar. 2004. 